\title{
Antimitogenic effects of HDL and APOE mediated by Cox-2-dependent IP activation
}

\author{
Devashish Kothapalli, ${ }_{1}^{1}$ Ilia Fuki, ${ }^{2}$ Kamilah Ali, ${ }^{2}$ Sheryl A. Stewart,${ }^{1}$ Liang Zhao, ${ }^{3}$ \\ Ron Yahil, ${ }^{3}$ David Kwiatkowski, ${ }^{3}$ Elizabeth A. Hawthorne, ${ }^{1}$ Garret A. FitzGerald, ${ }^{1}$ \\ Michael C. Phillips, ${ }^{4}$ Sissel Lund-Katz, ${ }^{4}$ Ellen Puré, $, 3,5$ Daniel J. Rader, ${ }^{2}$ \\ and Richard K. Assoian ${ }^{1}$ \\ ${ }^{1}$ Department of Pharmacology, and
${ }^{2}$ Department of Medicine, University of Pennsylvania School of Medicine, Philadelphia, Pennsylvania, USA
${ }^{3}$ The Wistar Institute, Philadelphia, Pennsylvania, USA
${ }^{4}$ The Children's Hospital of Philadelphia Abramson Research Center, University of Pennsylvania School of Medicine,
Philadelphia, Pennsylvania, USA
${ }^{5}$ The Ludwig Institute for Cancer Research, New York, New York, USA
}

HDL and its associated apo, APOE, inhibit S-phase entry of murine aortic smooth muscle cells. We report here that the antimitogenic effect of APOE maps to the $\mathrm{N}$-terminal receptor-binding domain, that APOE and its $\mathrm{N}$-terminal domain inhibit activation of the cyclin A promoter, and that these effects involve both pocket protein-dependent and independent pathways. These antimitogenic effects closely resemble those seen in response to activation of the prostacyclin receptor IP. Indeed, we found that HDL and APOE suppress aortic smooth muscle cell cycle progression by stimulating Cox-2 expression, leading to prostacyclin synthesis and an IP-dependent inhibition of the cyclin A gene. Similar results were detected in human aortic smooth muscle cells and in vivo using mice overexpressing APOE. Our results identify the Cox-2 gene as a target of APOE signaling, link HDL and APOE to IP action, and describe a potential new basis for the cardioprotective effect of HDL and APOE.

J. Clin. Invest. 113:609-618 (2004). doi:10.1172/JCI200419097.

\section{Introduction}

APOE is a 34-kDa protein associated with triglyceriderich lipoproteins and HDL. It plays an important role in preventing atherosclerotic disease and is a major determinant of susceptibility to human coronary artery disease (1). APOE is composed of an N-terminal domain that contains a LDL receptor-binding region and a $\mathrm{C}$-terminal domain that possesses a lipid-binding region. Many studies have indicated that APOE inhibits atherosclerosis in part by reducing plasma cholesterol through hepatic clearance of remnant lipoproteins and cholesterol-rich HDL (1). Recent reports have suggested, however, that APOE may also protect against atherosclerosis by mechanisms independent of its ability to modulate plasma lipid levels (1-5), and some of these studies show that APOE inhibits smooth muscle proliferation and

Received for publication June 2, 2003, and accepted in revised form December 16, 2003.

Address correspondence to: Richard K. Assoian, 167 Johnson Pavilion, 3620 Hamilton Walk, Department of Pharmacology, University of Pennsylvania School of Medicine, Philadelphia, Pennsylvania 19104-6084, USA. Phone: (215) 898-7157; Fax: (215) 573-5656; E-mail: rka@pharm.med.upenn.edu. Conflict of interest: The authors have declared that no conflict of interest exists.

Nonstandard abbreviations used: smooth muscle cells (SMCs); cyclin-dependent kinase (cdk); retinoblastoma protein ( $\mathrm{pRb}$ ); cAMP response element (CRE); cAMP response element-binding protein (CREB); electrophoretic mobility shift assay (EMSA); 6-keto-prostaglandin $\mathrm{F}_{1 \alpha}$ (6-keto-PGF $\mathrm{PG}_{1 \alpha}$ ); recombinant adenovirus encoding the human APOE3 cDNA (AdAPOE3).
G1-phase cycle progression $(4,5)$. Zhu et al. have demonstrated that APOE inhibits medial smooth muscle cell (SMC) proliferation after vascular injury in vivo (6).

G1-phase cell-cycle progression is regulated by a sequential activation of G1-phase cyclin and cyclindependent kinase (cdk) complexes, namely cyclin D1cdk4 and cyclin E-cdk2. Active cyclin D1-cdk4/6 and cyclin E-cdk2 complexes regulate G1-phase progression in part by phosphorylating the retinoblastoma protein (pRb) and the related pocket proteins p107 and p130 (7). Phosphorylation of the pocket proteins by G1-phase cyclin-cdk complexes leads to the transcription of E2Fresponsive genes, such as cyclin A. E2F4-p107 transcriptionally represses the cyclin A gene in early G1-phase, and E2F1-3 transcriptionally activates the gene at G1/S (8). Other studies, however, show that E2F-pocket protein-independent mechanisms are also required for efficient induction of cyclin A transcription; for example the cAMP-response element (CRE) is necessary for the efficient induction of the cyclin $A$ gene $(9,10)$. Ultimately, the expression of cyclin A and the consequent formation of cyclin A-cdk2 complexes marks entry into S-phase of the cell cycle.

Proliferation of aortic SMCs plays a clear role in restenosis, and many studies indicate that it also plays a role in vascular remodeling and atherosclerosis. Likely regulators of SMC proliferation in vivo include TGF- $\beta$, prostacyclin, and NO, all of which are released from endothelial cells and are thought to have an antimitogenic effect on the neighboring SMCs in the normal ves- 
sel $(11,12)$. We have recently studied the cell-cycle effects of prostacyclin in early-passage murine aortic SMCs and found that it inhibits progression into S-phase by blocking both the phosphorylation of pocket proteins and the binding of cAMP response element-binding protein (CREB)/phosphoCREB to the cyclin A promoter (13). As expected, these effects were mediated by IP, the prostacyclin receptor. Furthermore, IP deletion in mice results in an increased proliferative response to injury (14).

In this study, we have investigated the mechanism by which HDL and APOE inhibit the proliferation of aortic SMCs. We show that both HDL and APOE block S-phase entry and cyclin $A$ gene expression and that the resulting G1-phase arrest closely resembles that observed upon IP activation. We also show that HDL and APOE induce Cox-2 gene expression, that the inhibitory effects of APOE on S-phase entry are reversed by a Cox-2-specific inhibitor, and that IP is required for the antimitogenic effect of APOE. Thus, the antimitogenic effects of HDL and APOE are mediated by the induction of Cox-2 and a Cox-2-dependent synthesis of prostacyclin. Overall, these results provide a new basis for thinking about the cardioprotective effects of HDL and APOE.

\section{Methods}

Cell culture. Primary murine aortic SMCs were isolated from WT C57BL/6 or IP-null mice, maintained as described (15), and used between passage 2 and 5. Purity of the SMC population was confirmed by the expression of $\alpha$-smooth muscle actin as detected by immunofluorescence (not shown). Near-confluent monolayers of early-passage murine aortic SMCs or rat A10 SMCs (American Type Culture Collection, Manassas, Virginia, USA) were G0 synchronized by 2-day incubation in serum-free defined medium (13). The quiescent cells were stimulated with $10 \%$ FBS in the absence or presence of LDL, HDL, APOE-depleted HDL, lipid-free human APOA-I, lipid-free human APOE3 (the major isoform of human APOE; hereafter called APOE), the N-terminal domain of APOE (APOE22), or the C-terminal domain of APOE (APOE10). APOE-depleted HDL was prepared by passing HDL over a heparin-Sepharose column (16). The recombinant APOA-I, APOE, APOE22, and APOE10 were expressed and purified as previously described (16, 17). Human aortic SMCs (Cambrex Bio Science Walkersville Inc., Walkersville, Maryland, USA) and aortic endothelial cells (a generous gift of Elliot Levine, The Wistar Institute, Philadelphia, Pennsylvania, USA) were maintained as described $(18,19)$.

Immunofluorescence. Murine aortic SMCs were grown to $80-90 \%$ confluence in $35-\mathrm{mm}$ dishes containing autoclaved glass coverslips, were serum starved, and were stimulated with $10 \%$ FBS in the absence or presence of lipoproteins or apo's as outlined above. Fixed cells were analyzed by immunofluorescence for incorporation of BrdU or expression of cyclin A as described (13). The percentage of BrdU-positive cells was assessed by counting the number of FITC-stained cells relative to DAPI-stained nuclei using epifluorescence microscopy (100-200 cells were counted from several fields of view).

Immunoblotting. Aortic SMCs or A10 SMCs were lysed as described (20), and total protein concentration was determined by Coomassie binding (Bio-Rad Laboratories Inc., Hercules, California, USA). Equal amounts of protein $(50 \mu \mathrm{g})$ were fractionated and analyzed by immunoblotting as described previously (20) using Ab's specific for the following proteins: phospho-CREB (05-667), CREB (06-863) (both from Upstate Biotechnology Inc., Lake Placid, New York, USA); pRb (28-0007) (Zymed Laboratories Inc., South San Francisco, California, USA); cyclin D1 (sc-8396), cyclin E (sc-481), p107 (sc-318), ATF-1 (sc-243), actin (sc-8432), and cdk4 (sc-260) (all from Santa Cruz Biotechnology Inc., Santa Cruz, California, USA); and Cox-2 (BD Biosciences, San Jose, California, USA). Rabbit polyclonal cyclin A Ab was prepared in this laboratory using recombinant cyclin $A$ as an immunogen. Cell transfections and promoter analyses. Near-confluent (80-90\%) murine aortic SMCs in 35- $\mathrm{mm}$ dishes were transiently transfected with $+\mathrm{CRE}$ and -CRE cyclin A pro- $\mathbf{a}$

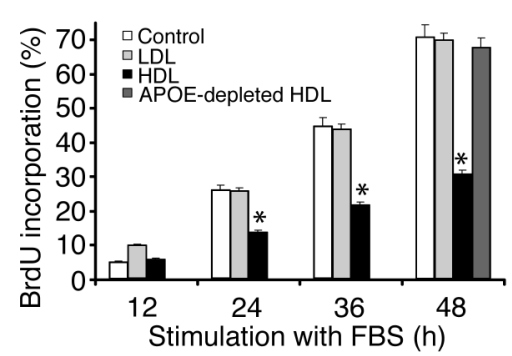

b

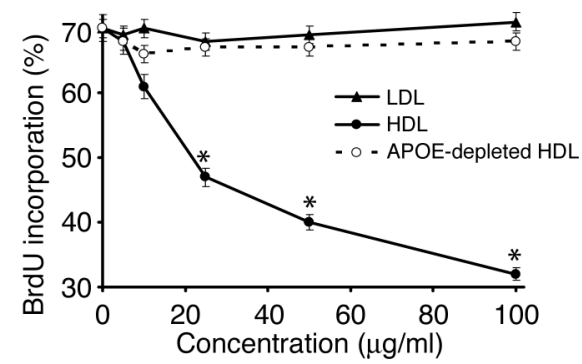

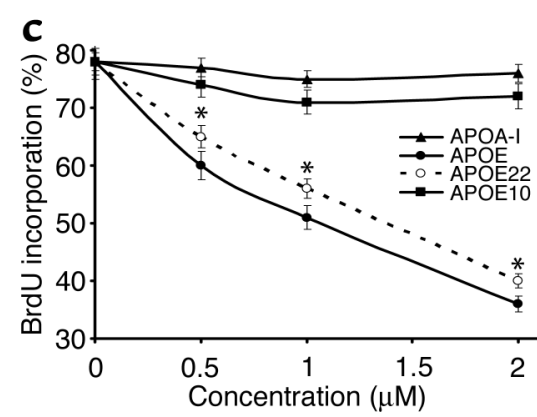

Figure 1

HDL and APOE inhibit S-phase entry in aortic SMCs. (a) Quiescent aortic SMCs were trypsinized and replated with $10 \%$ FBS for selected times in the absence (control) or presence of $50 \mu \mathrm{g} / \mathrm{ml} \mathrm{LDL,} \mathrm{HDL,} \mathrm{or} \mathrm{APOE-depleted} \mathrm{HDL.} \mathrm{(b)} \mathrm{Quiescent} \mathrm{cells} \mathrm{were} \mathrm{stimulated} \mathrm{with} 10 \%$ FBS for 48 hours in the presence of increasing concentrations of LDL, HDL, or APOE-deficient HDL. (c) Quiescent aortic SMCs were trypsinized, added to $35-\mathrm{mm}$ dishes containing coverslips, and stimulated with $10 \%$ FBS for 48 hours in the presence of increasing concentrations of APOA-I, APOE, APOE22, or APOE10. BrdU incorporation into nuclei was determined by immunofluorescence microscopy. Results show the mean $\pm \mathrm{SEM}, n=3,{ }^{*} P<0.01$ as compared with cells treated with $10 \% \mathrm{FBS}$ alone. 


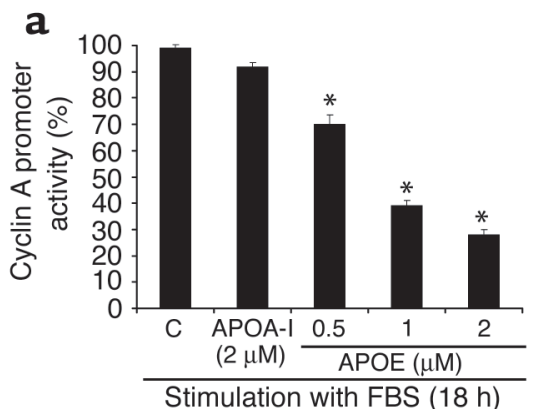

C

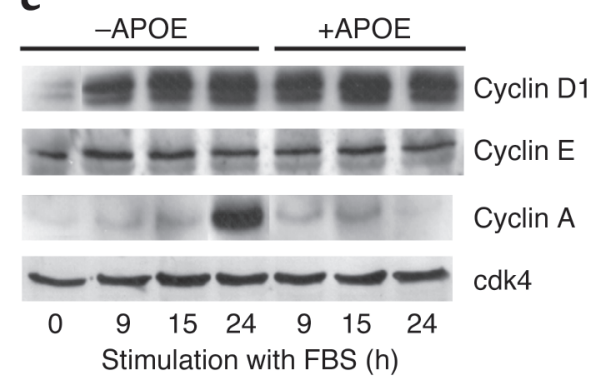

b
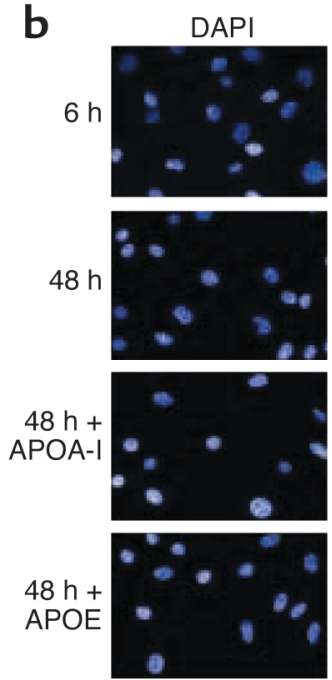

Cyclin A
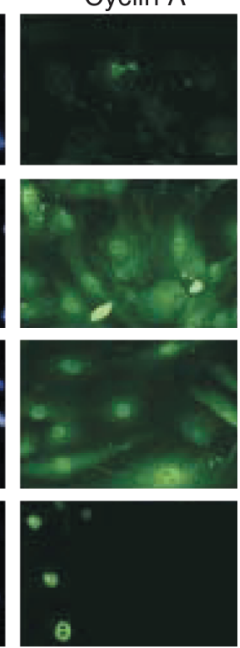

d

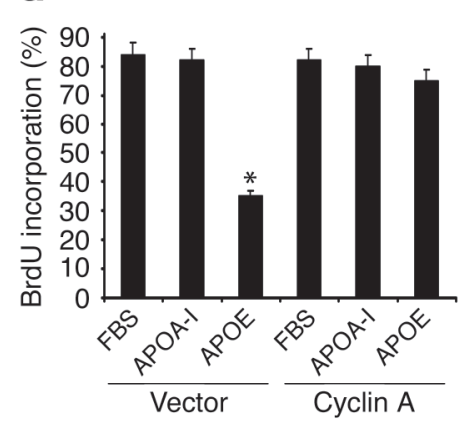

e

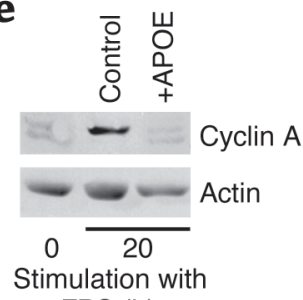

FBS (h)

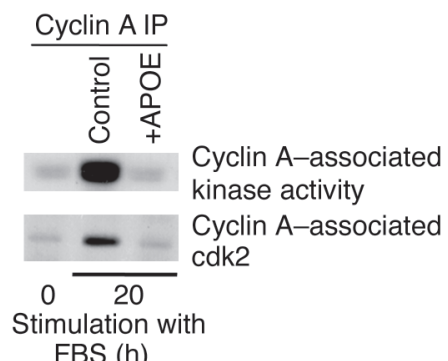

FBS (h)

\section{Figure 2}

Inhibition of cyclin A gene expression by APOE. (a) Cyclin A promoter activity in early-passage aortic SMCs was determined as described in Methods. Results show mean $\pm \mathrm{SEM}, n=3,{ }^{*} P<0.001$ as compared with cells stimulated with $10 \% \mathrm{FBS}$. C, control. (b) Quiescent SMCs were incubated for 48 hours with 10\% FBS in the absence or presence of $2 \mu \mathrm{M}$ APOA-I or APOE prior to analysis of cyclin A expression by immunofluorescence microscopy. DAPI staining shows cell nuclei. (c) Quiescent murine aortic SMCs treated with 10\% FBS in the absence or presence of $2 \mu \mathrm{M}$ APOE were collected, lysed, and analyzed by immunoblotting using Ab's to cyclin D1, cyclin E, cyclin A, and cdk4 (loading control). (d) Aortic SMCs were transiently cotransfected with an eGFP expression vector and either a control (vector) or cyclin A expression vector. The effect of cyclin A expression on S-phase entry was determined in the absence or presence of $2 \mu \mathrm{M}$ APOE or $2 \mu \mathrm{M}$ APOA-I as described in Methods. Results show mean $\pm \mathrm{SEM}, n=2,{ }^{*} P<0.001$ as compared with cells stimulated with $10 \% \mathrm{FBS}$ (vector). (e) A10 SMCs were treated as in c, collected at 20 hours, lysed, and analyzed by immunoblotting for cyclin A and actin (loading control). Cell lysates were incubated with anticyclin A, and the immunoprecipitates were used to assess in vitro kinase activity or analyzed by immunoblotting for associated cdk 2 .

moter-luciferase vectors (13) using $5 \mu$ l of Lipofectamine (Invitrogen, Carlsbad, California, USA), $5 \mu$ Lipofectamine Plus reagent (Invitrogen), $1 \mu \mathrm{g}$ cyclin A promoterluciferase plasmid(s), and either $1 \mu \mathrm{g}$ of a human papilloma virus type-18 E7 expression vector or $1 \mu \mathrm{g}$ of empty vector. The transfections also included $0.1 \mu \mathrm{g}$ of a Renillaluciferase expression plasmid (pRL-CMV; Promega Corp., Madison, Wisconsin, USA) to control for transfection efficiency. Transfection efficiency obtained in these cells was $25-30 \%$ as determined by transient transfection of an eGFP expression vector. After an overnight recovery, the cells were serum starved for 24 hours and directly stimulated with $10 \%$ FBS for 18 hours in the presence of $2 \mu \mathrm{M}$ APOE, APOA-I, APOE22, or APOE10, or $10 \mathrm{ng} / \mathrm{ml}$ TGF- $\beta 1$. Cyclin A promoter-driven luciferase activity was determined and normalized to Renilla-luciferase (13).

Transient transfections of murine aortic SMCs with a minimal E2F-CAT expression vector (21) used $1 \mu \mathrm{g}$ of the E2F-CAT plasmid and either $1 \mu \mathrm{g}$ of the human papilloma virus type $18 \mathrm{E} 7$ expression vector or $1 \mu \mathrm{g}$ of empty vector. Cells were washed with PBS and collected. CAT levels were measured from duplicate samples using an enzyme immunoassay kit (Roche Applied Science, Indianapolis, Indiana, USA).

The cyclin A expression vector was created by subcloning the 1.5-kb EcoR1-XhoI fragment of human cyclin A cDNA into pCMV-Tag 5A (Stratagene, La Jolla, California, USA). Murine aortic SMCs were grown to $80-90 \%$ confluence in $35-\mathrm{mm}$ dishes containing autoclaved glass coverslips. The cells were transiently cotransfected with $0.1 \mu \mathrm{g}$ eGFP expression vector and $1 \mu \mathrm{g}$ pCMV-Tag $5 \mathrm{~A}$ or $1 \mu \mathrm{g}$ cyclin $\mathrm{A}$ expression vector. After an overnight recovery, the cells were serum starved for 24 hours and then directly stimulated with $10 \%$ FBS for 24 hours in the presence of $2 \mu \mathrm{M}$ APOE or $2 \mu \mathrm{M}$ APOA-I. S-phase entry of the transfected (eGFP-positive) cells was determined by BrdU incorporation. 


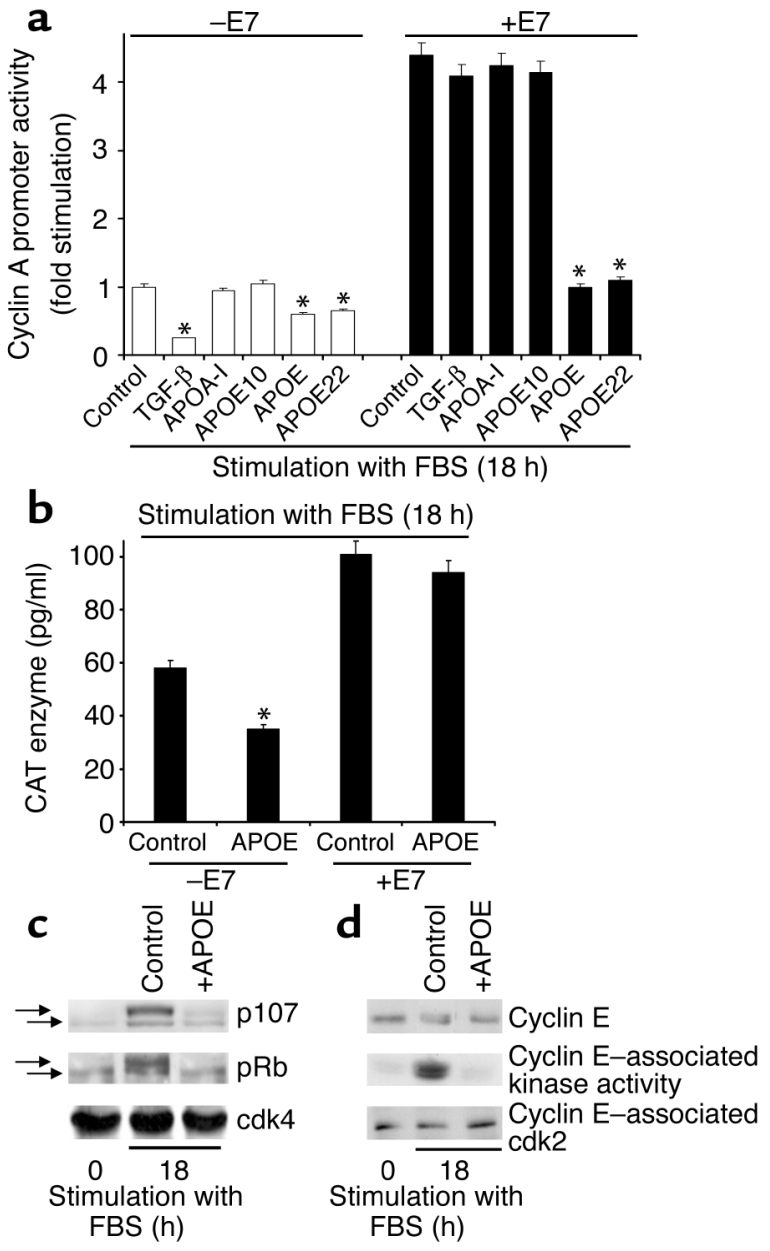

For electrophoretic mobility shift assays (EMSAs) and super-shift assays, murine aortic SMCs were brought to $80-90 \%$ confluence in $150-\mathrm{mm}$ dishes, serum starved, and directly stimulated for 18 hours with $10 \%$ FBS in the absence or presence of APOA-I or APOE. Nuclear lysates were prepared and incubated with labeled oligonucleotides corresponding to the CRE and CCAAT site as described (13).

In vitro kinase assays. A10 SMCs were grown to 80-90\% confluence in 150-mm dishes, serum starved, and stimulated with $10 \%$ FCS in the absence or presence of $2 \mu \mathrm{M}$ APOE as outlined above. Cells were washed once with PBS and lysed as described (22). Extracts from collected cells were incubated with anti-cyclin $\mathrm{E}(4 \mu \mathrm{g} / 300 \mu \mathrm{g}$ lysate $)$ or an ammonium sulfate fraction of anti-cyclin A $(40 \mu \mathrm{g} / 100 \mu \mathrm{g}$ lysate). Collected immunoprecipitates were divided into two equal fractions and used to measure kinase activity toward histone $\mathrm{H} 1$ or complex formation with $\mathrm{cdk} 2$ as described (13).

The 6-keto-prostaglandin $F_{1 \alpha}$ enzyme immunoassay. Quiescent WT and IP-null SMCs were brought to $80-90 \%$ confluence in $100-\mathrm{mm}$ dishes, serum starved, and directly stimulated with $10 \%$ FBS in the absence or presence of $2 \mu \mathrm{M}$ APOE. Conditioned media $(100 \mu \mathrm{l})$ were collected from the cells after 24 or 48 hours. The

\section{Figure 3}

Pocket protein-dependent effects of APOE on cyclin A gene transcription. (a) Cyclin A promoter activity in early-passage aortic SMCs was determined as described in Methods. Promoter activity is plotted as fold stimulation relative to the FBS-treated cells in the absence of E7. Results show the mean \pm SEM, $n=3,{ }^{*} P<0.001$ as compared with transfected cells stimulated with FBS alone. (b) Early-passage aortic SMCs were transiently cotransfected with the E2F-CAT plasmid and either the E7 expression vector (+E7) or empty vector (-E7). After serum starvation, the quiescent transfectants were directly stimulated with $10 \%$ FBS for 18 hours in the absence (control) or presence of $2 \mu \mathrm{M}$ APOE. CAT enzyme levels were measured as described in Methods. Results show the mean $\pm \mathrm{SEM}, n=2,{ }^{*} P<0.001$ as compared with transfected cells stimulated with FBS alone (control; -E7). (c) Quiescent mouse aortic SMCs were serum stimulated in the absence or presence of $2 \mu \mathrm{M}$ APOE for 18 hours. Collected cells were lysed and analyzed by immunoblotting for pRb, p107, and cdk4. The hypophosphorylated and hyperphosphorylated forms of pRb and p107 are shown by lower and upper arrows, respectively. (d) Quiescent A10 SMCs were serum stimulated in the absence or presence of $2 \mu \mathrm{M}$ APOE for 15 hours, collected, lysed, and analyzed by immunoblotting for cyclin E. Cell lysates were incubated with anti-cyclin E, and the immunoprecipitates were used to assess in vitro kinase activity or were analyzed by immunoblotting for associated cdk2.

conditioned media were then assayed for 6-ketoprostaglandin $\mathrm{F}_{1 \alpha}$ (6-keto-PGF $\left.\mathrm{F}_{1 \alpha}\right)$ using an enzyme immunoassay kit (Cayman Chemical, Ann Arbor, Michigan, USA).

Analysis of Cox-2 mRNA and protein. The effects of lipoproteins and apo's on Cox-2 mRNA and protein were determined by PCR and immunoblotting, respectively. Murine aortic SMCs were grown to $80-90 \%$ confluence in 150-mm dishes, serum starved for 48 hours in defined medium (see above), and directly stimulated with $10 \%$ FBS for 18 hours. Total RNA was isolated from cells lysed in Trizol reagent (Invitrogen), and $2 \mu \mathrm{g}$ from each sample was used for cDNA synthesis using the Superscript II preamplification system (Invitrogen). An aliquot (10\%) of cDNA was then subjected to 35 cycles of PCR using Taq DNA polymerase, melting at $94^{\circ} \mathrm{C}$, annealing at $55^{\circ} \mathrm{C}(1$ minute), and extending at $72^{\circ} \mathrm{C}(1$ minute). The primers were specific for mouse Cox-1, Cox-2, and GAPDH (23) or human Cox-1 (sense: 5'-CTGGTGGATGCCTTCTCTCGCC-3'; antisense: $5^{\prime}$ GTCTTGGTGTTGAGGCAGACCAG-3'), Cox-2 (sense: $5^{\prime}$ CAGCAAATCCTTGCTGTTCC-3'; antisense: 5'-GAGAAGGCTTCCCAGCTTT- $3^{\prime}$ ). The amplified products were analyzed by agarose gel electrophoresis and ethidium bromide staining, and changes in abundance were quantified using NIH Image 1.63 software. Lysates of aortic SMCs were also analyzed by immunoblotting for Cox-2 protein as described above.

In vivo stimulation of Cox- 2 mRNA by APOE expression. To express APOE in vivo, a second-generation recombinant adenovirus encoding the human APOE3 cDNA (AdAPOE3) was used as previously described (24). A total of 12, 6- to 8-week-old, female, WT C57BL/6 mice (four per group) were injected in the tail vein with $10^{11}$ particles of either AdAPOE3, a second- 
a

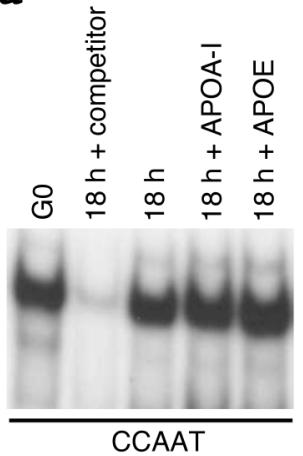

C

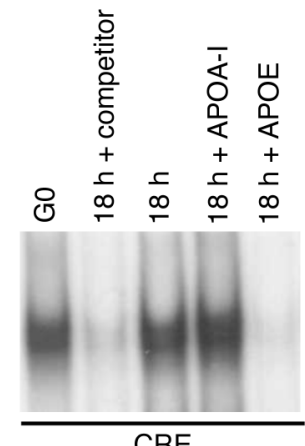

CRE

b
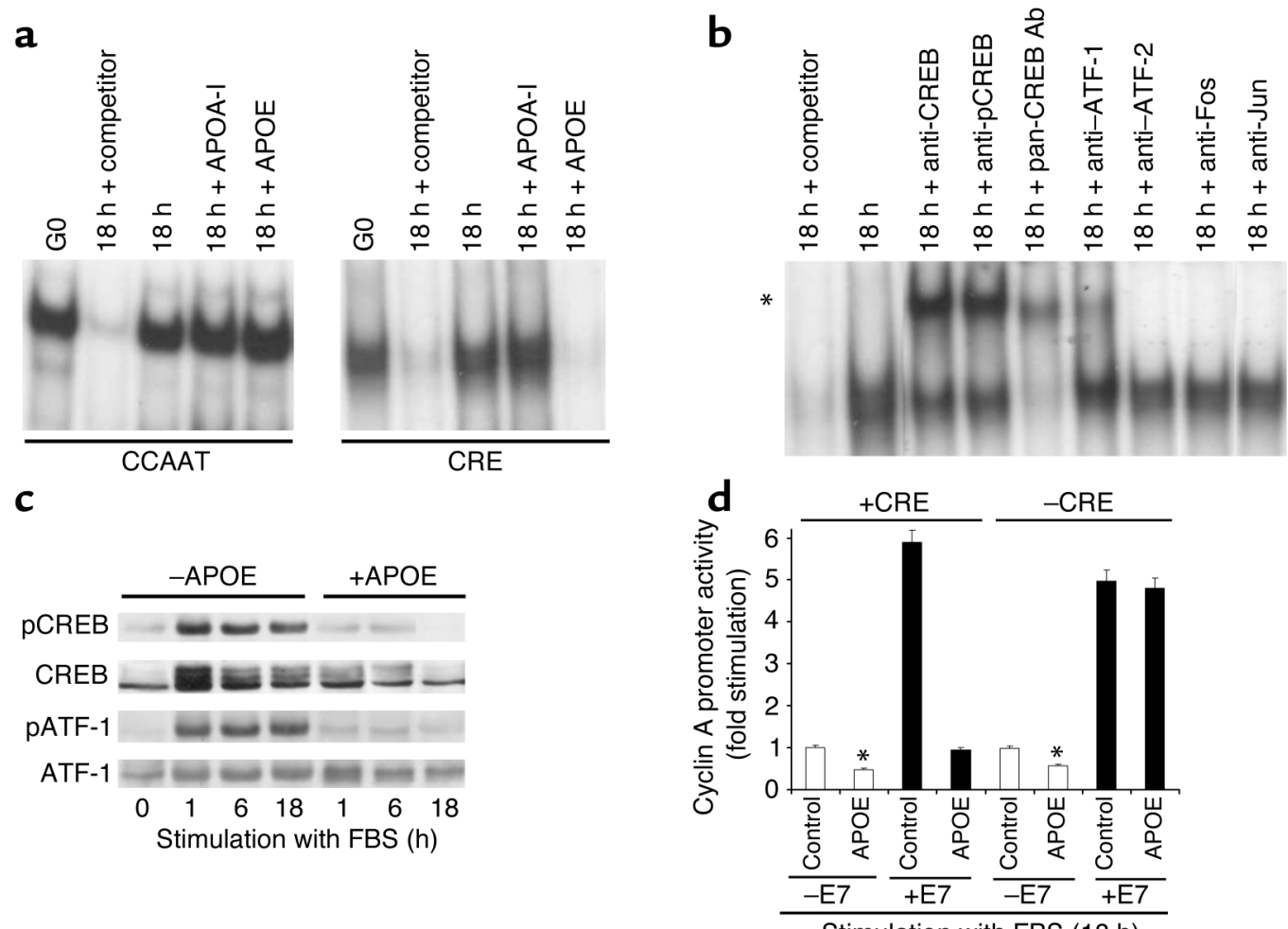

Stimulation with FBS (18 h)

\section{Figure 4}

CRE-dependent effects of APOE on cyclin A gene transcription. (a) EMSAs were performed with ${ }^{32}$ P-labeled CCAAT or CRE oligonucleotides and nuclear lysates from serum-starved SMCs before (G0) or after stimulation with $10 \%$ FBS for 18 hours in the absence or presence of APOE or APOA-I. Competitor, 100-fold molar excess unlabeled oligonucleotide. (b) Nuclear extracts were prepared from serum-starved SMCs after 18 -hour incubation with $10 \%$ FBS. The extracts were preincubated with the indicated Ab's before addition of the ${ }^{32} \mathrm{P}$-labeled CRE oligonucleotide. ${ }^{*}$ Supershifted complex. (c) Serum-starved murine aortic SMCs were incubated with $10 \%$ FBS in the absence or presence of APOE. Cells were collected, lysed, and analyzed by immunoblotting using anti-phosphoCREB, anti-CREB, and anti-ATF-1. Note that the anti-phosphoCREB Ab detects both phosphoCREB (pCREB) and phosphoATF-1 (pATF-1). (d) Activity of the +CRE or -CRE cyclin A promoters was determined in the absence (-E7) or presence (+E7) of E7 and the absence (control) or presence of $2 \mu M$ APOE. Promoter activity is plotted as fold stimulation relative to the FBS-treated cells in the absence of E7. Results show the mean \pm SEM, $n=3,{ }^{*} P<0.001$ as compared with vector-transfected cells (-E7) stimulated with FBS (+CRE and -CRE). Normalized luciferase activities of the cyclin A promoters containing and lacking the CRE in control (FBS-stimulated) cells were 0.05 and 0.02 , respectively.

generation AdAPOA-I virus, or control virus (Adnull). Mice were sacrificed 3 days after injection and perfused with ice-cold PBS, and the entire aorta was harvested. Our previous studies showed that the adenovirus-expressed proteins accumulated within the vessel wall (25). Aortae were dissected, and extraneous tissue from the adventitial side was carefully removed. The tissue was then immediately submerged into RNAlater (QIAGEN Inc., Valencia, California, USA) and manually homogenized. The homogenate was treated with proteinase $\mathrm{K}\left(55^{\circ} \mathrm{C}, 10\right.$ minutes; QIAGEN Inc.) and clarified by centrifugation $\left(30\right.$ minutes, $\left.10,000 \mathrm{~g}, 20-25^{\circ} \mathrm{C}\right)$. The supernatant was mixed with $0.5 \times$ volume of ethanol and purified on an RNeasy mini column (QIAGEN Inc.). Total RNA $(2 \mu \mathrm{g})$ was reverse transcribed using the Superscript II preamplification system. An aliquot $(40 \%)$ of the cDNA was analyzed by PCR as outlined above. Procedures involving mice were approved by the University of Pennsylvania and Wistar Institutional Animal Care and Use Committees.

\section{Results}

HDL and APOE inhibit S-phase entry in aortic SMCs. We initially compared the effects of HDL and LDL on S-phase entry of early-passage aortic SMCs. Aortic SMCs were serum starved and stimulated with serum at subconfluence in the presence of BrdU; these cells showed a timedependent increase into S-phase during 48 hours of serum stimulation. LDL had a negligible effect on the kinetics of S-phase entry, but HDL inhibited S-phase entry more than 50\% (Figure 1a). Moreover, the antimitogenic effect of HDL was lost when APOE was depleted from the lipoprotein (Figure 1a). The effect of HDL was dose dependent, with an $\mathrm{IC}_{50}$ of approximately $20 \mu \mathrm{g} / \mathrm{ml}$ (Figure 1b). Purified recombinant APOE also inhibited S-phase entry in a dose-dependent manner while purified recombinant APOA-I had no effect (Figure 1c). We produced the $22-\mathrm{kDa} \mathrm{N}$-terminal and $10-\mathrm{kDa}$ C-terminal domains of APOE as recombinant proteins and found that the antimitogenic activity of APOE was largely contained within the $\mathrm{N}$-terminal receptor-binding domain (Figure 1c; compare APOE10 versus APOE22). 
APOE inbibits cyclin A gene expression via pocket proteindependent and independent pathways. Since the induction of cyclin A regulates entry of cells into S-phase, the effect of APOE on cyclin A expression and promoter activity was examined in early-passage aortic SMCs. We compared the effects of APOE and APOA-I on aortic SMCs transiently transfected with a cyclin A promoter-luciferase reporter expression vector. Initial studies showed that the cyclin A promoter was optimally induced approximately 18 hours after serum stimulation of quiescent SMCs (data not shown). APOA-I $(2 \mu \mathrm{M})$ minimally $(<10 \%)$ affected activation of the cyclin A promoter, whereas APOE showed a dose-dependent inhibition that reached greater than $70 \%$ (Figure 2a). Cyclin A was expressed in $85 \%$ of nuclei after aortic SMCs were stimulated with serum or serum with APOA-I (Figure 2b). Cyclin A was expressed in only 35\% of nuclei in the cells treated with serum and APOE (Figure $2 \mathrm{~b}$ ), however. A time-course analysis showed that cyclin A expression was induced in aortic SMCs by 24 hours of serum stimulation and that the induction was inhibited by APOE (Figure 2c). Cyclin D1 and cyclin E were not affected by APOE (Figure 2c). Moreover, ectopic expression of cyclin A in mouse SMCs rescued S-phase entry in the presence of APOE (Figure 2d). APOE also inhibited S-phase entry (not shown), cyclin A expression, and cyclin A-associated kinase activity in the rat A10 SMC line (Figure 2e).

We cotransfected murine aortic SMCs with a cyclin A promoter-luciferase reporter construct and a human papilloma virus type 18 E7 expression vector to study the effect of APOE on pocket protein-dependent cyclin A promoter activity. E7 sequesters pocket proteins (26) and mimics cdk-mediated phosphorylation, resulting in transcription of genes such as cyclin A. The transfected cells were serum starved and then serum stimulated for 18 hours in the presence of APOA-I, APOE, or the isolated APOE22 (N terminal) and APOE10 (C terminal) domains. Cells were also treated with TGF- $\beta$, an antimitogen that acts largely by inhibiting pocket protein phosphorylation (27). As shown in Figure 3a, E7 caused cyclin A promoter activity to increase more than fourfold in cells treated with serum, APOA-I, the C-terminal domain of APOE, or TGF- $\beta$, presumably due to complete pocket protein sequestration and release of E2F. Although E7 also stimulated cyclin A promoter activity in the cells treated with APOE or its N-terminal domain (APOE22), the effect was small relative to that seen with the other treatments. Since APOE and its $\mathrm{N}$-terminal domain inhibit the cyclin A promoter despite pocket protein sequestration by E7, they either (a) inhibit the promoter via a pocket protein-independent pathway or (b) have pocket protein-independent as well as -dependent effects on the cyclin A promoter. Similar results were obtained using APOE or the N-terminal domain with the A10 SMC line (not shown).

We cotransfected murine aortic SMCs with a minimal E2F-CAT promoter-reporter construct and the E7 expression vector to determine if the effect of APOE on cyclin A promoter activity involves an $\mathrm{E} 2 \mathrm{~F} /$ pocket protein-dependent pathway. The transfected cells were serum starved and then stimulated with serum for 18 hours in the presence or absence of APOE. APOE inhibited E2F activity (Figure 3b; -E7), and the inhibition of E2F activity was fully overcome by the expression of E7 (Figure 3b; +E7). Thus, APOE may inhibit E2F-dependent genes in general. Moreover, APOE blocked the hyperphosphorylation of $\mathrm{pRb}$ and $\mathrm{p} 107$ in both murine aortic SMCs (Figure 3c) and the A10 SMC line (not shown). This effect was associated with the inhibition of cyclin E-cdk2 activity (Figure 3d), but the exact mechanism is

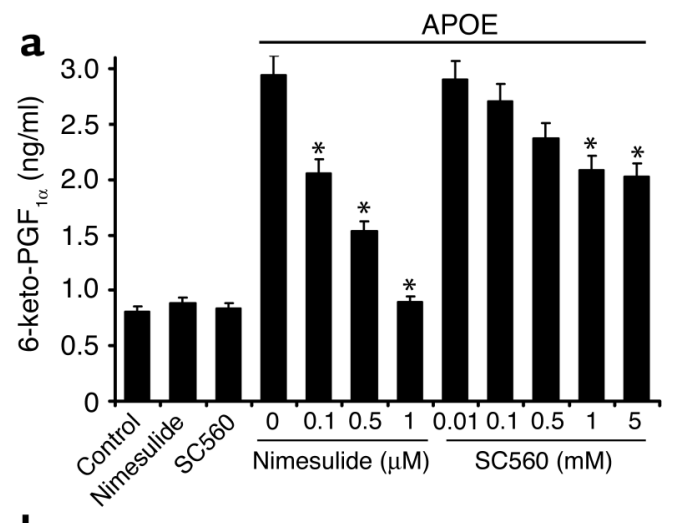

b

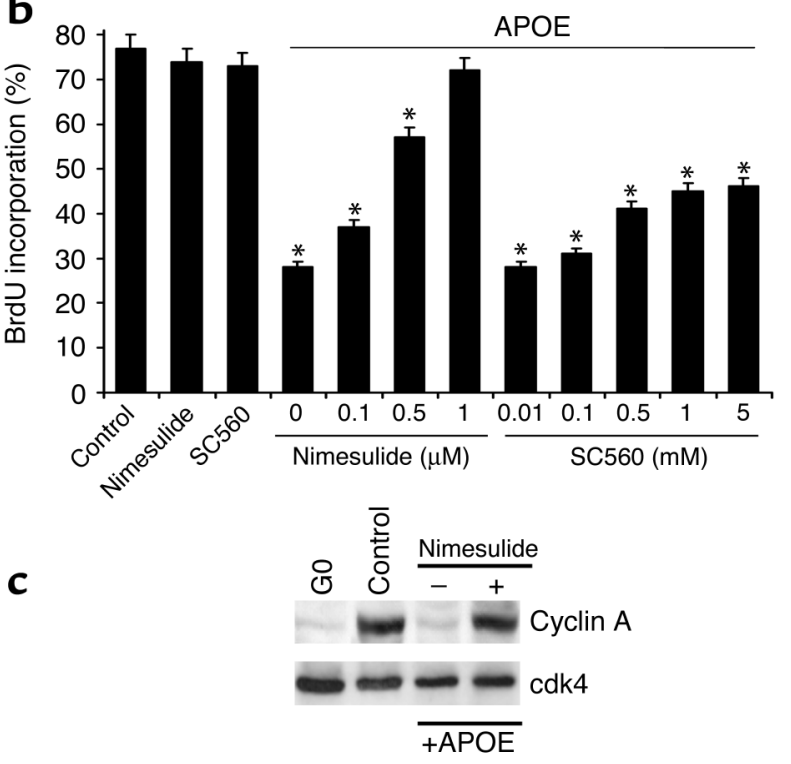

Figure 5

The antimitogenic effect of APOE requires Cox-2. Murine aortic SMCs were serum starved and treated with $10 \%$ FBS in the absence (control) or presence of $2 \mu \mathrm{M}$ APOE. Cells were also treated with $0.1-1 \mu \mathrm{M}$ nimesulide or $0.01-5 \mathrm{mM} \mathrm{SC560}$. All the cells were incubated for 48 hours in the presence of BrdU. (a) The conditioned media was collected and assayed for 6-keto-PGF $1 \alpha$. Results show mean $\pm \mathrm{SEM}, n=2$, ${ }^{*} P<0.005$ as compared with cells treated with $10 \%$ FBS (control) and $2 \mu \mathrm{M}$ APOE. (b) BrdU incorporation into nuclei was determined by immunofluorescence microscopy. Results show mean $\pm \mathrm{SEM}, n=2$, ${ }^{*} P<0.005$ as compared with cells treated with $10 \%$ FBS (control). (c) Quiescent murine aortic SMCs were treated with 10\% FBS for 24 hours in the absence (control) or presence of APOE and $0.5 \mu \mathrm{M}$ nimesulide. Cells were collected, lysed, and analyzed by immunoblotting for cyclin A and cdk4 (loading control). 

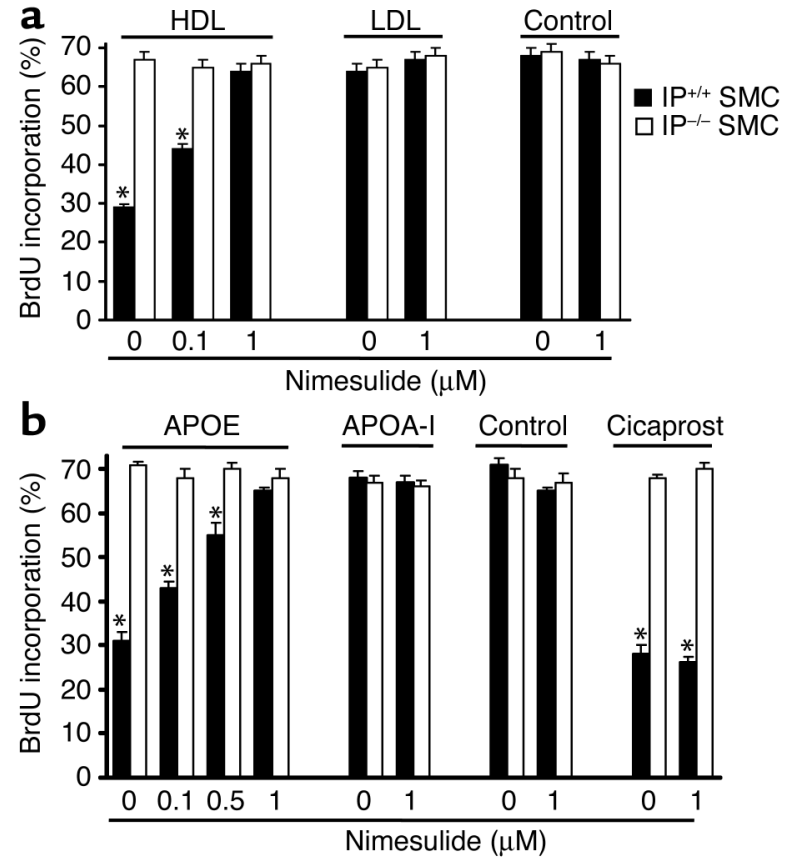

C

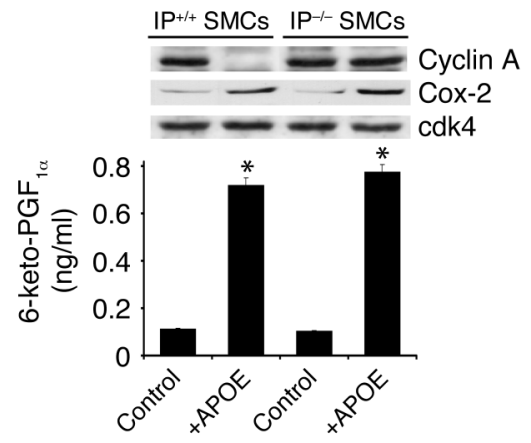

not yet clear since APOE did not alter the levels of cyclin D1, cyclin E, cdk2, cdk4, p21 cip1, or p27kip1 (Figure 3, c-d, and data not shown). Interestingly, this is the same phenotype we saw when examining the antimitogenic effect of prostacyclin (ref. 13 and see below).

APOE inhibits CRE-dependent cyclin A gene expression. Since the results described in Figure 3, b-d, indicate that APOE should inhibit E2F/pocket protein-dependent transcription, the absence of strong cyclin A promoter activity in APOE-treated cells expressing E7 (Figure 3a) was likely due to additional, pocket protein-independent effects of APOE on the cyclin A promoter. Indeed, EMSAs showed that while APOE did not alter occupancy of the cyclin A promoter CCAAT site, it strongly decreased occupancy of its CRE site (Figure 4a). In contrast, APOA-I affected neither site (Figure 4a). Supershift EMSAs showed that CREB and a small amount of ATF-1 were bound to the CRE, but other potential CRE-binding proteins, such as ATF-2, $c$-fos, and c-jun, were not detected (Figure $4 b$ ). Note that a pan-CREB family Ab fully shifted the CRE complex (Figure 4b) and that CREM (which comprises the CREB family along with CREB and ATF-1) is not expressed in aortic SMCs (28). These results indicate

\section{Figure 6}

Inhibition of S-phase entry by APOE is dependent on Cox-2 and IP. (a and b) Aortic SMCs isolated from WT (black bars) and IP-null (white bars) mice were cultured, serum starved for 48 hours, and treated with $10 \%$ FBS in the absence (control) or presence of $\mathrm{HDL}(50 \mu \mathrm{g} / \mathrm{ml})$, LDL $(50 \mu \mathrm{g} / \mathrm{ml})$, APOE $(2 \mu \mathrm{M})$,or APOA-I $(2 \mu \mathrm{M})$. Cells were also treated with $0.1-1 \mu \mathrm{M}$ nimesulide or $200 \mathrm{nM}$ cicaprost, a prostacyclin mimetic (13). All the cells were incubated for 48 hours in the presence of BrdU. BrdU incorporation into nuclei was determined by immunofluorescence microscopy. Results show the mean \pm SEM, $n=3,{ }^{*} P<0.001$ as compared with cells treated with $10 \%$ FBS (control). (c) Aortic SMCs from WT and IP-null mice were serum starved and treated with $10 \%$ FBS for 24 hours in the absence (control) or presence of $2 \mu \mathrm{M}$ APOE. Conditioned media was then collected and assayed for 6-keto-PGF $1 \alpha$, while the cell lysates were analyzed by immunoblotting using anti-cyclin A, anti-Cox-2, and anti-cdk4 (loading control). The bar graph in $\mathbf{c}$ shows mean $\pm \mathrm{SEM}, n=2,{ }^{*} P<0.001$ as compared with cells treated with $10 \%$ FBS (control).

that CREB, ATF-1, and their phosphorylated counterparts can fully account for the CRE-binding activity of the SMC lysate. Overall, these results suggested that APOE-dependent inhibition of CRE occupancy and CREB/ATF-1 phosphorylation could account for the pocket protein-independent effect of APOE on cyclin A gene expression. Indeed, we found that APOE also inhibits serum-induced phosphorylation of CREB and ATF-1 throughout the G1-phase (Figure 4c).

To assess the composite effects of APOE on CRE- and pocket protein-dependent cyclin $A$ gene expression, we transiently cotransfected early-passage aortic SMCs with the $\mathrm{E} 7$ expression vector and cyclin A promoter-luciferase reporter constructs containing (+CRE) or lacking (-CRE) the CRE element (Figure 4d). The transfectants were serum starved and then stimulated with serum in the absence or presence of APOE. In the absence of E7, APOE inhibited luciferase activity whether or not the CRE was present in the cyclin A promoter. In the presence of E7, however, APOE inhibited luciferase activity only when the promoter contained the CRE. These results demonstrate that the combined effects of APOE on E2F/pocket protein- and CRE-dependent transcription can fully account for its inhibitory effect on the cyclin A promoter.

The antimitogenic effect of APOE is mediated by a Cox-2dependent activation of IP. The effects of APOE on cyclin E-cdk2 activity and cyclin A gene expression closely resembled those we observed when studying the antimitogenic effect of IP activation in aortic SMCs (13). Moreover, HDL has been reported to induce Cox-2, and Cox-2 expression can lead to prostacyclin synthesis and activation of IP (29). Consistent with this report, we found that the Cox-2 selective inhibitor, nimesulide, strongly inhibited prostacyclin biosynthesis (measured as 6-keto-PGF ${ }_{1 \alpha}$, a stable metabolite of prostacyclin) induced by APOE, while a selective Cox-1 inhibitor, SC560, had a much weaker effect (Figure 5a). We therefore reasoned that the antimitogenic effects of HDL and APOE might result from stimulation of Cox-2 gene expression, synthesis of prostacyclin, and activation of IP. Indeed, we found that a nonselective Cox inhibitor (indomethacin; data not 
shown) and nimesulide (Figure $5 \mathrm{~b}$ ) completely reversed the antimitogenic effect of APOE in murine aortic SMCs whereas the effect of SC560 was relatively small (Figure $5 \mathrm{~b})$. Nimesulide also restored the expression of cyclin A in APOE-treated murine aortic SMCs (Figure 5c). Moreover, we found that nimesulide blocked the antimitogenic effect of HDL (Figure 6a; black bars) in early-passage aortic SMCs. This effect was specific since S-phase entry in response to serum, LDL, or APOA-I was unaffected by nimesulide (Figure 6 , a and b, black bars). Finally, nimesulide did not reverse the antimitogenic effect of cicaprost (a prostacyclin mimetic), which activates IP downstream of Cox-2 (Figure 6b, black bars).

The experiments in Figure 6, a and b, were also performed with early-passage aortic SMCs isolated from IPnull mice to directly implicate IP in the antimitogenic effect of APOE. Neither HDL nor APOE were antimitogenic in IP-null SMCs (Figures 6, a and b; white bars). Moreover, while APOE had similar stimulatory effects on Cox-2 expression and prostacyclin biosynthesis (Figure $6 \mathrm{c}$ ) in $\mathrm{IP}^{+/+}$and $\mathrm{IP}^{-/-}$SMCs, it failed to inhibit cyclin A expression in the IP-null cells (Figure 6c). Taken together, the results indicate that an APOE-dependent induction of Cox-2 leads to increased prostacyclin synthesis, IP activation, and the pocket protein-dependent and-independent antimitogenic effects outlined above. We directly examined the effect of HDL, LDL, APOE, and APOA-I on Cox- 1 and Cox- 2 expression in SMCs. RT-PCR (Figure 7a) of RNA isolated from early-passage murine SMCs showed that both HDL and APOE induced Cox- 2 mRNA (3.5-fold) over control, while LDL and APOA-I had no effect. None of these treatments affected Cox-1 mRNA (Figure 7a). Immunoblotting of total cell lysates showed that APOE, but not APOA-I, also increased the expression of Cox-2 protein in murine SMCs (Figure 7b). Importantly, the effects of APOE that we observed in cultured murine SMCs are also detected in cultured human SMCs: APOE induced Cox- 2 mRNA and protein (Figure 7, c and d, respectively) while the effect of APOA-I was either not detectable (Figure $7 \mathrm{c}$ ) or quite small (Figure $7 \mathrm{~d}$ ). The increase in Cox-2 expression is cell-type specific since APOE did not alter Cox-2 levels in cultured human aortic endothelial cells (Figure 7d). This cell type-specific response to APOE allowed us to explore the potential effect of APOE on vascular Cox-2 mRNA levels in vivo by harvesting aortae from mice overexpressing APOE or APOA-I. APOE specifically increased Cox-2 mRNA levels in freshly isolated aortae (Figure 7e). Thus, the stimulatory effect of APOE on Cox-2 gene expression occurs both in cell culture and in vivo.

\section{Discussion}

The results described above demonstrate that APOE mediates the antimitogenic effect of HDL on inhibition of S-phase entry and that the molecular effects of APOE on CREB, the G1-phase cyclin-cdk's, and CRE- and E2F-dependent cyclin $A$ gene expression are essentially indistinguishable from those seen upon activation of IP. We also show that (a) specific inhibition of Cox-2 blocks the antimitogenic effects of HDL and APOE, (b) both HDL and APOE induce Cox-2 gene expression, and (c) IP is required for the antimitogenic effects of HDL and APOE. Taken together, these data indicate that APOE mediates the antimitogenic effects of HDL by the induction of Cox-2, prostacyclin-dependent activation of IP, and consequent inhibition of CRE- and pocket proteindependent cyclin A gene expression. The salient results have been reproduced in both murine and human SMC cultures and in vivo using overexpression of APOE in mice.

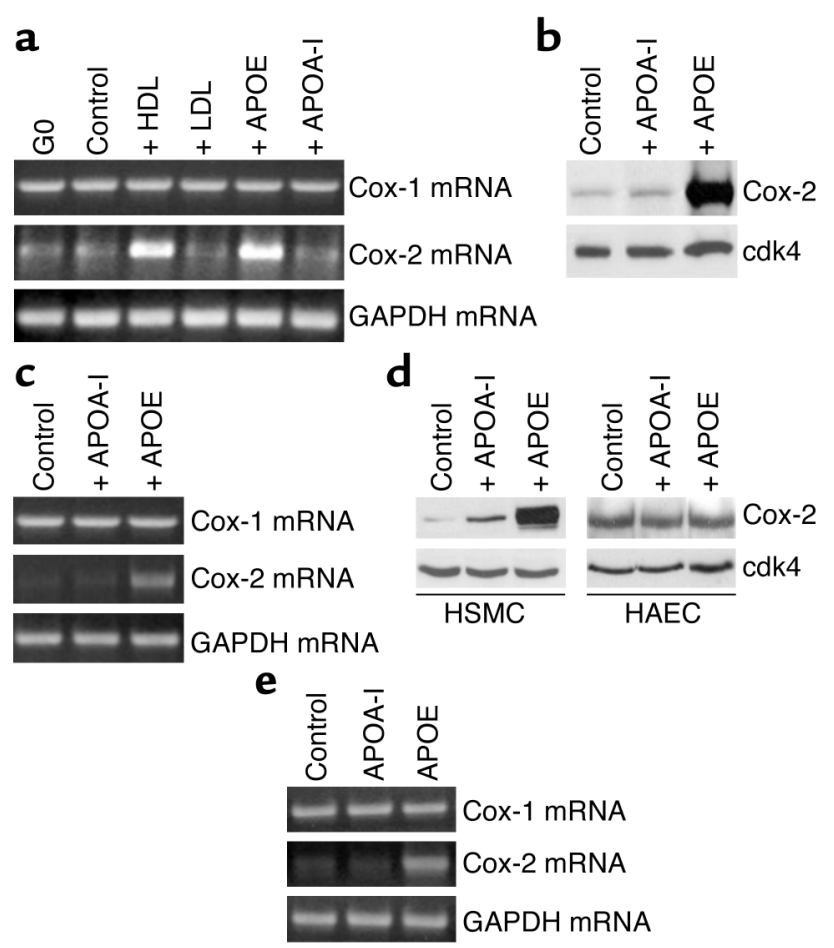

\section{Figure 7}

Conserved effects of APOE on Cox-2 expression in human and mouse SMCs and mice. (a) Quiescent mouse aortic SMCs were treated with $10 \%$ FBS in the absence or presence of $50 \mu \mathrm{g} / \mathrm{ml} \mathrm{HDL}, 50$ $\mu \mathrm{g} / \mathrm{ml} \mathrm{LDL}, 2 \mu \mathrm{M}$ APOE or $2 \mu \mathrm{M}$ APOA-I. Changes in the levels of Cox-1, Cox-2, and GAPDH (loading control) mRNAs were determined by RT-PCR and analyzed using NIH Image 1.63 software. (b) Cell lysates made from serum-starved aortic SMCs stimulated with $10 \% \mathrm{FBS}$ in the absence and presence of $2 \mu \mathrm{M}$ APOA-I or APOE were immunoblotted using anti-Cox-2 and anti-cdk4 (loading control). Human aortic SMCs (HSMC) were grown to near confluence in $150-\mathrm{mm}$ dishes, serum starved for 48 hours in defined medium, and then directly stimulated with $10 \%$ FBS in the absence or presence of $2 \mu \mathrm{M}$ APOE or $2 \mu \mathrm{M}$ APOA-I. Changes in the levels of Cox- 2 mRNA (c) and protein (d) were determined as in $\mathbf{a}$ and $\mathbf{b}$, respectively. Cell lysates were also made from serum-starved human aortic endothelial cells (HAEC) stimulated with $2 \mu \mathrm{M}$ APOA-I or APOE, and the levels of Cox-2 protein were determined by immunoblotting (d). (e) C57BL/ 6 mice were injected with adenoviral constructs expressing APOA-I, APOE, or null vector. Total RNA was isolated from the aortae and reverse transcribed into cDNA. Changes in the levels of Cox-1, Cox-2, and GAPDH mRNA were determined as outlined in Methods. 
Ishigami et al. have reported that APOE inhibits PDGF-mediated S-phase entry by inducing iNOS (30) and inhibiting the expression of cyclin D1 mRNA (4). In our studies, however, APOE did not affect cyclin D1. Moreover, NOS inhibitors (L-NAME and AG, nonselective and NOS2-selective inhibitors, respectively) did not prevent the antimitogenic effect of APOE even though they effectively reversed the antimitogenic effect of the NO donor SNP (data not shown). Other studies have indicated that the antimitogenic effect of APOE involves heparan sulfate proteoglycans and that the C-terminal heparin-binding domain of APOE binds to heparan sulfate proteoglycan $(2,5)$. In contrast, we find that the antimitogenic effect of APOE is contained within the $\mathrm{N}$-terminal receptor-binding domain of APOE. Although the basis for these apparently discrepant results is not completely clear, it may well reflect our use of a more broad-based mitogen (serum) rather than PDGF or bFGF $(2,5)$; the mitogenic effects of these growth factors require heparan sulfate proteoglycans as coreceptors (31).

Mammalian Cox exists as two isoforms, Cox- 1 and Cox-2 (32). These isoforms are produced by separate genes but are structurally homologous (33). Others have reported that Cox-2 levels and prostacyclin production are increased by HDL in aortic SMCs and endothelial cells $(34,35)$, and the results shown here indicate that this effect of HDL is mediated by APOE. Interestingly, Cox- 2 production has been linked to inhibition of cell proliferation in several different cell types, including myofibroblasts, fibroblasts, SMCs, and epithelial cells $(14,36,37)$. It is important to emphasize, however, that Cox-2 has also been associated with stimulation of cell proliferation and transformation in epithelial cells (38, 39). Thus, there are likely to be diverse consequences of Cox-2 production depending on the cell type-specific expression of downstream syntheses.

Increasing evidence demonstrates that Cox-2 expression is not limited to sites of inflammation, but that it is differentially regulated in various cell types $(40,41)$ and plays an important role in vascular homeostasis (42). Prostacyclin, a dominant product of Cox-2 in humans (43), is the major Cox product of macrovascular endothelium (44). It is thought to protect against arterial thrombosis by modulating the biological effects of thromboxane $\mathrm{A} 2$ on platelets and vascular function (14). In addition to its effects on platelet function and vascular tone (45), prostacyclin also modulates the release of tissue plasminogen inhibitor (46) and cholesterol efflux (47). The results shown here indicate that both HDL and APOE can exert antimitogenic effects on vascular SMCs via Cox-2 and activation of IP-dependent signaling pathways. This novel mechanism may well contribute to the antiatherogenic effects of HDL and APOE and could lead to new approaches for the prevention of cardiovascular disease. Suppression of this HDL effect might also be a part of the spectrum of cardiovascular hazards presented by Cox- 2 inhibitors $(14,48,49)$.

\section{Acknowledgments}

We thank William Fahl (University of Wisconsin) for the cyclin A promoter-luciferase constructs, Lou Laimins (Northwestern University) for the HPV type 18 E7 expression vector, and Kevin Chow (Washington University) for the E2F-CAT vector. This work was supported by NIH grant HL-62250 and the Commonwealth Universal Research Enhancement Program, Pennsylvania Department of Health. D. Kothapalli was supported by an American Heart Association postdoctoral fellowship (20160U). D.J. Rader is a recipient of a Burroughs Wellcome Fund Clinical Scientist Award in Translational Research and is a Doris Duke Distinguished Clinical Scientist. G.A. FitzGerald is the Robinette Professor of Cardiovascular Medicine and the Elmer Bobst Professor of Pharmacology.

1. Mahley, R.W. 1988. Apolipoprotein E: cholesterol transport protein with expanding role in cell biology. Science. 240:622-630.

2. Swertfeger, D.K., and Hui, D.Y. 2001. Apolipoprotein E receptor binding versus heparan sulfate proteoglycan binding in its regulation of smooth muscle cell migration and proliferation. J. Biol. Chem. 276:25043-25048.

3.Ho, Y.Y., Deckelbaum, R.J., Chen, Y., Vogel, T., and Talmage, D.A. 2001. Apolipoprotein E inhibits serum-stimulated cell proliferation and enhances serum-independent cell proliferation. J. Biol. Chem. 276:43455-43462.

4. Ishigami, M., Swertfeger, D.K., Granholm, N.A., and Hui, D.Y. 1998. Apolipoprotein E inhibits platelet-derived growth factor-induced vascular smooth muscle cell migration and proliferation by suppressing signal transduction and preventing cell entry to G1 phase. J. Biol. Chem. 273:20156-20161.

5. Paka, L., et al. 1999. Perlecan mediates the antiproliferative effect of apolipoprotein $\mathrm{E}$ on smooth muscle cells. An underlying mechanism for the modulation of smooth muscle cell growth? J. Biol. Chem. 274:36403-36408.

6. Zhu, B., Kuhel, D.G., Witte, D.P., and Hui, D.Y. 2000. Apolipoprotein E inhibits neointimal hyperplasia after arterial injury in mice. Am. J. Pathol. 157:1839-1848.

7. Classon, M., and Dyson, N. 2001. p107 and p130: versatile proteins with interesting pockets. Exp. Cell. Res. 264:135-147.

8. Takahashi, Y., Rayman, J.B., and Dynlacht, B.D. 2000. Analysis of promoter binding by the E2F and $\mathrm{PRB}$ families in vivo: distinct E2F proteins mediate activation and repression. Genes Dev. 14:804-816.

9. Bottazzi, M.E., et al. 2001. Distinct effects of mitogens and the actin cytoskeleton on CREB and pocket protein phosphorylation control the extent and timing of cyclin A promoter activity. Mol. Cell. Biol. 21:7607-7616.

10. Desdouets, C., et al. 1995. Cell cycle regulation of cyclin A gene expression by the cyclic AMP-responsive transcription factors CREB and CREM. Mol. Cell. Biol. 15:3301-3309.

11. Topper, J.N., Cai, J., Falb, D., and Gimbrone, M.A., Jr. 1996. Identification of vascular endothelial genes differentially responsive to fluid mechanical stimuli: cyclooxygenase-2, manganese superoxide dismutase, and endothelial cell nitric oxide synthase are selectively up-regulated by steady laminar shear stress. Proc. Natl. Acad. Sci. U. S. A. 93:10417-10422.

12. Cucina, A., et al. 1998. Shear stress induces transforming growth factorbeta 1 release by arterial endothelial cells. Surgery. 123:212-217.

13. Kothapalli, D., et al. 2003. Prostacylin receptor activation inhibits proliferation of aortic smooth muscle cells by regulating cAMP response element-binding protein- and pocket protein-dependent cyclin a gene expression. Mol. Pharmacol. 64:249-258.

14. Cheng, Y., et al. 2002. Role of prostacyclin in the cardiovascular response to thromboxane A2. Science. 296:539-541.

15. Cuff, C.A., et al. 2001. The adhesion receptor CD44 promotes atherosclerosis by mediating inflammatory cell recruitment and vascular cell activation. J. Clin. Invest. 108:1031-1040. doi:10.1172/JCI200112455.

16. Gillotte, K.L., et al. 1999. Apolipoprotein-mediated plasma membrane microsolubilization. Role of lipid affinity and membrane penetration in the efflux of cellular cholesterol and phospholipid. J. Biol. Chem. 274:2021-2028.

17. Saito, H., et al. 2001. Lipid binding-induced conformational change in human apolipoprotein E. Evidence for two lipid-bound states on spherical particles. J. Biol. Chem. 276:40949-40954.

18. Motamed, K., et al. 2002. Inhibition of PDGF-stimulated and matrixmediated proliferation of human vascular smooth muscle cells by 
SPARC is independent of changes in cell shape or cyclin-dependent kinase inhibitors. J. Cell. Biochem. 84:759-771.

19. Thornton, S.C., Mueller, S.N., and Levine, E.M. 1983. Human endothelial cells: use of heparin in cloning and long-term serial cultivation. Science. 222:623-625.

20. Roovers, K., Davey, G., Zhu, X., Bottazzi, M.E., and Assoian, R.K. 1999 Alpha5beta1 integrin controls cyclin D1 expression by sustaining mitogen-activated protein kinase activity in growth factor-treated cells. Mol. Biol. Cell. 10:3197-3204.

21. Loeken, M.R., and Brady, J. 1989. The adenovirus EIIA enhancer. Analysis of regulatory sequences and changes in binding activity of ATF and EIIF following adenovirus infection. J. Biol. Chem. 264:6572-6579.

22. Zhu, X., Ohtsubo, M., Bohmer, R.M., Roberts, J.M., and Assoian, R.K. 1996. Adhesion-dependent cell cycle progression linked to the expression of cyclin D1, activation of cyclin E-cdk2, and phosphorylation of the retinoblastoma protein. J. Cell. Biol. 133:391-403.

23. Rocca, B., et al. 1999. Distinct roles of prostaglandin H synthases 1 and 2 in T-cell development. J. Clin. Invest. 103:1469-1477.

24. Tangirala, R.K., et al. 2001. Reduction of isoprostanes and regression of advanced atherosclerosis by apolipoprotein E. J. Biol. Chem. 276:261-266.

25. Tsukamoto, K., Tangirala, R., Chun, S.H., Pure, E., and Rader, D.J. 1999. Rapid regression of atherosclerosis induced by liver-directed gene transfer of ApoE in ApoE-deficient mice. Arterioscler. Thromb. Vasc. Biol. 19:2162-2170

26. Vousden, K. 1993. Interactions of human papillomavirus transforming proteins with the products of tumor suppressor genes. FASEB J. 7:872-879.

27. Reynisdottir, I., Polyak, K., Iavarone, A., and Massague, J. 1995. Kip/Cip and Ink $4 \mathrm{Cdk}$ inhibitors cooperate to induce cell cycle arrest in response to TGF-beta. Genes Dev. 9:1831-1845.

28. Palermo, I., et al. 2001. Cloning and expression of activator of CREM in testis in human testicular tissue. Biochem. Biophys. Res. Commun. 283:406-411.

29. Vinals, M., Martinez-Gonzalez, J., and Badimon, L. 1999. Regulatory effects of HDL on smooth muscle cell prostacyclin release. Arterioscler. Thromb. Vasc. Biol. 19:2405-2411.

30. Ishigami, M., Swertfeger, D.K., Hui, M.S., Granholm, N.A., and Hui, D.Y. 2000. Apolipoprotein E inhibition of vascular smooth muscle cell proliferation but not the inhibition of migration is mediated through activation of inducible nitric oxide synthase. Arterioscler. Thromb. Vasc. Biol. 20:1020-1026.

31. Silver, P.J., et al. 1998. Heparinase III limits rat arterial smooth muscle cell proliferation in vitro and in vivo. Eur. J. Pharmacol. 351:79-83.

32. Hla, T., and Neilson, K. 1992. Human cyclooxygenase-2 cDNA. Proc. Natl. Acad. Sci. U. S. A. 89:7384-7388.

33. Kosaka, T., et al. 1994. Characterization of the human gene (PTGS2) encoding prostaglandin-endoperoxide synthase 2. Eur. J. Biochem. 221:889-897.
34. Pomerantz, K.B., Tall, A.R., Feinmark, S.J., and Cannon, P.J. 1984. Stimulation of vascular smooth muscle cell prostacyclin and prostaglandin E2 synthesis by plasma high and low density lipoproteins. Circ. Res. 54:554-565.

35. Vinals, M., Martinez-Gonzalez, J., Badimon, J.J., and Badimon, L. 1997. HDL-induced prostacyclin release in smooth muscle cells is dependent on cyclooxygenase-2 (Cox-2). Arterioscler. Thromb. Vasc. Biol. 17:3481-3488.

36. Davaille, J., et al. 2000. Antiproliferative properties of sphingosine 1phosphate in human hepatic myofibroblasts. A cyclooxygenase- 2 mediated pathway. J. Biol. Chem. 275:34628-34633.

37. Lama, V., Moore, B.B., Christensen, P., Toews, G.B., and Peters-Golden, M. 2002. Prostaglandin E2 synthesis and suppression of fibroblast proliferation by alveolar epithelial cells is cyclooxygenase-2-dependent. Am. J. Respir. Cell Mol. Biol. 27:752-758.

38. Herschman, H.R. 1994. Regulation of prostaglandin synthase-1 and prostaglandin synthase-2. Cancer Metastasis Rev. 13:241-256.

39. Ko, S.C., et al. 2002. Paracrine cyclooxygenase-2-mediated signalling by macrophages promotes tumorigenic progression of intestinal epithelial cells. Oncogene. 21:7175-7186.

40. Vadlamudi, R., et al. 1999. Regulation of cyclooxygenase-2 pathway by HER2 receptor. Oncogene. 18:305-314.

41. Hull, M.A., et al. 1999. Cyclooxygenase 2 is up-regulated and localized to macrophages in the intestine of Min mice. Br. J. Cancer. 79:1399-1405.

42. Dubois, R.N., et al. 1998. Cyclooxygenase in biology and disease. FASEBJ. 12:1063-1073.

43. McAdam, B.F., et al. 1999. Systemic biosynthesis of prostacyclin by cyclooxygenase (COX)-2: the human pharmacology of a selective inhibitor of COX-2. Proc. Natl. Acad. Sci. U. S. A. 96:272-277.

44. Moncada, S., and Vane, J.R. 1981. Prostacyclin and the vascular endothelium. Bull. Eur. Physiopathol. Respir. 17:687-701.

45. Fitzgerald, G.A., Catella, F., and Oates, J.A. 1987. Eicosanoid biosynthesis in human cardiovascular disease. Hum. Pathol. 18:248-252.

46. Hegeman, R.J., van den Eijnden-Schrauwen, Y., and Emeis, J.J. 1998. Adenosine $3^{\prime}: 5^{\prime}$-cyclic monophosphate induces regulated secretion of tissue-type plasminogen activator and von Willebrand factor from cultured human endothelial cells. Thromb. Haemost. 79:853-858.

47. Pomerantz, K.B., and Hajjar, D.P. 1990. High-density-lipoproteininduced cholesterol efflux from arterial smooth muscle cell derived foam cells: functional relationship of the cholesteryl ester cycle and eicosanoid biosynthesis. Biochemistry. 29:1892-1899.

48. Bombardier, C., et al. 2000. Comparison of upper gastrointestinal toxicity of rofecoxib and naproxen in patients with rheumatoid arthritis VIGOR Study Group. N. Engl. J. Med. 343:1520-1528.

49. FitzGerald, G.A. 2003. COX-2 and beyond: approaches to prostaglandin inhibition in human disease. Nat. Rev. Drug Discov. 2:879-890. 